Kompass

Ophthalmologie

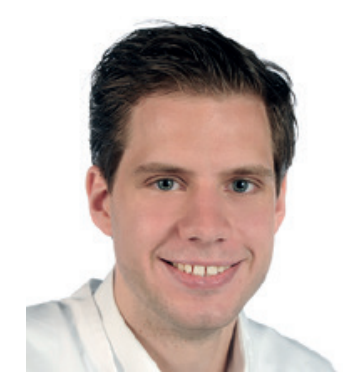

\title{
Prognostic factors for visual acuity in pseudophakic patients with epiretinal gliosis and macular surgery
}

Maximilian Treder

Klinik für Augenheilkunde, Universitätsklinikum Münster, Münster, Germany

Abstract of Kim JY, Kim DY, Kim KT, et al.: Visual prognostic factors of epiretinal membrane surgery in patients with pseudophakia. Ophthalmologica 2019;DOI:10.1159/000502748.

\section{Keywords}

Epiretinal membrane · Optical coherence tomography

Pseudophakia · Visual prognosis · Vitrectomy

\begin{abstract}
Purpose: To evaluate the visual prognostic factors in patients with pseudophakic epiretinal membrane (ERM) after vitrectomy using spectral domain optical coherence tomography (SD-OCT).

Methods: A retrospective review of patients with pseudophakic ERM having undergone vitrectomy was conducted. Best corrected visual acuity (BCVA) and SD-OCT were conducted before and 1,3 , and 6 months after vitrectomy. Known visual prognostic factors, such as inner-retina irregularity index, central foveal thickness (CFT), central inner retinal layer thickness (CIRLT), cone outer segment tip defect length, and photoreceptor outer segment length, were reviewed and their correlation with BCVA was analyzed.
\end{abstract}

Results: Forty-three patients (mean age: $64.88 \pm 10.46$ years) with pseudophakic ERM were included. BCVA significantly improved after vitrectomy (logMAR $0.30 \pm 0.24$ vs. $0.11 \pm 0.14, p<0.001)$. The preoperative high inner-retina irregularity index significantly correlated with poor postoperative BCVA in patients with pseudophakic ERM (correlation coefficient 0.583, $p<0.001$ ). Postoperative improvements of inner retinal SD-OCT findings, such as innerretina irregularity index, CFT, and CIRLT, were significantly associated with the amount of BCVA improvement after ERM surgery (correlation coefficients were as follows: inner-retina irregularity index - 0.711, $p<0.001$; CFT - 0.462, $p=0.002$; CIRLT $-0.596, p<$ 0.001). However, preoperative outer retinal SD-OCT findings were not associated with postoperative visual prognosis.

Conclusion: From this study, we determined the visual prognostic factors of ERM surgery without confounding factors, such as visual acuity improvement following combined cataract surgery, and inner retinal SD-OCT findings more significantly associated with the visual prognosis of ERM surgery compared to outer retinal SD-OCT findings.

(c) 2019 S. Karger AG, Basel 


\section{Knowledge transfer}

\section{Background}

Epiretinal gliosis is among the diseases of the vitreoretinal interface and can occur idiopathically or secondarily in connection with various clinical pictures, such as diabetic retinopathy or uveitis [1-3]. In idiopathic epiretinal gliosis, the incomplete posterior vitreous detachment leads to the formation of fibroproliferative, contractile membranes which morphologically lead to a distortion of the macula (Fig. 1) $[1,2,4]$. For the patient, this is associated with the symptoms of visual impairment and the perception of metamorphopsia $[1,2]$. Spectral domain optical coherence tomography (SD-OCT) is very well suited for visually representing the membrane and the associated changes and, thus, enables the tractive effect to be assessed [2-4]. In the case of epiretinal glioses, which have a tractive effect and which are associated with visual impairment, a pars plana vitrectomy with peeling of the epiretinal membrane is the treatment of choice $[1,2,4]$. The postoperative outcome depends on various factors.

\section{Results of the study}

In this connection, Kim et al. (2019) investigated the correlation between various pre- and postoperative SD-OCT parameters with regard to the prognostic significance for visual acuity in 43 eyes of 43 patients (Tab. 1). To minimise external influences on visual acuity (for example, due to cataract formation or cataract surgery), only patients with pre-existing pseudophakia were included in this study.

The results showed that changes to the inner layers of the retina have a significant influence on visual acuity. A postoperative improvement of the inner retina irregularity index, the central foveal thickness and the central inner retinal layer thickness was significantly associated with an improvement in visual acuity. A high preoperative inner retina irregularity index was additionally correlated with a worse postoperative visual acuity prognosis [5].

\section{Conclusions for clinical practice and perspectives for the future}

Taking objective SD-OCT parameters into account in the planning and indications for a vitrectomy with membrane peeling could, on

Table 1. SD-OCT parameters were investigated in connection with an epiretinal gliosis and its surgical treatment and their correlation with visual acuity in the study of Kim et al. [5]

Factors Definition

Inner-retina irregularity index Calculation: divide the length of the lower edge of the inner plexiform layer by the length of the retinal pigment epithelial cell layer

\begin{tabular}{ll}
\hline Central foveal thickness (CFT) & Central foveal layer thickness \\
\hline $\begin{array}{l}\text { Central inner retinal layer } \\
\text { thickness (CIRLT) }\end{array}$ & $\begin{array}{l}\text { Retinal nerve fibre layer + ganglion cell } \\
\text { layer + inner plexiform layer + inner } \\
\text { nuclear layer }\end{array}$ \\
\hline
\end{tabular}

Outer nuclear complex (ONC) Outer plexiform layer + outer nuclear thickness layer

Cone outer segment tips (COST) Defect length of the cone outer seg defect length ment tips

Photoreceptor outer segment Length of the outer segments of the (PROS) length photoceptors
Fig. 1. Preoperative $(\mathbf{a}, \mathbf{b})$ and postoperative ( $\mathbf{c}$, d) near infrared and SD-OCT image of a patient with epiretinal gliosis who had received a vitrectomy with membrane peeling. The images clearly show the improvements in the parameters listed in Table 1 despite the anatomy which continues to be significantly altered.
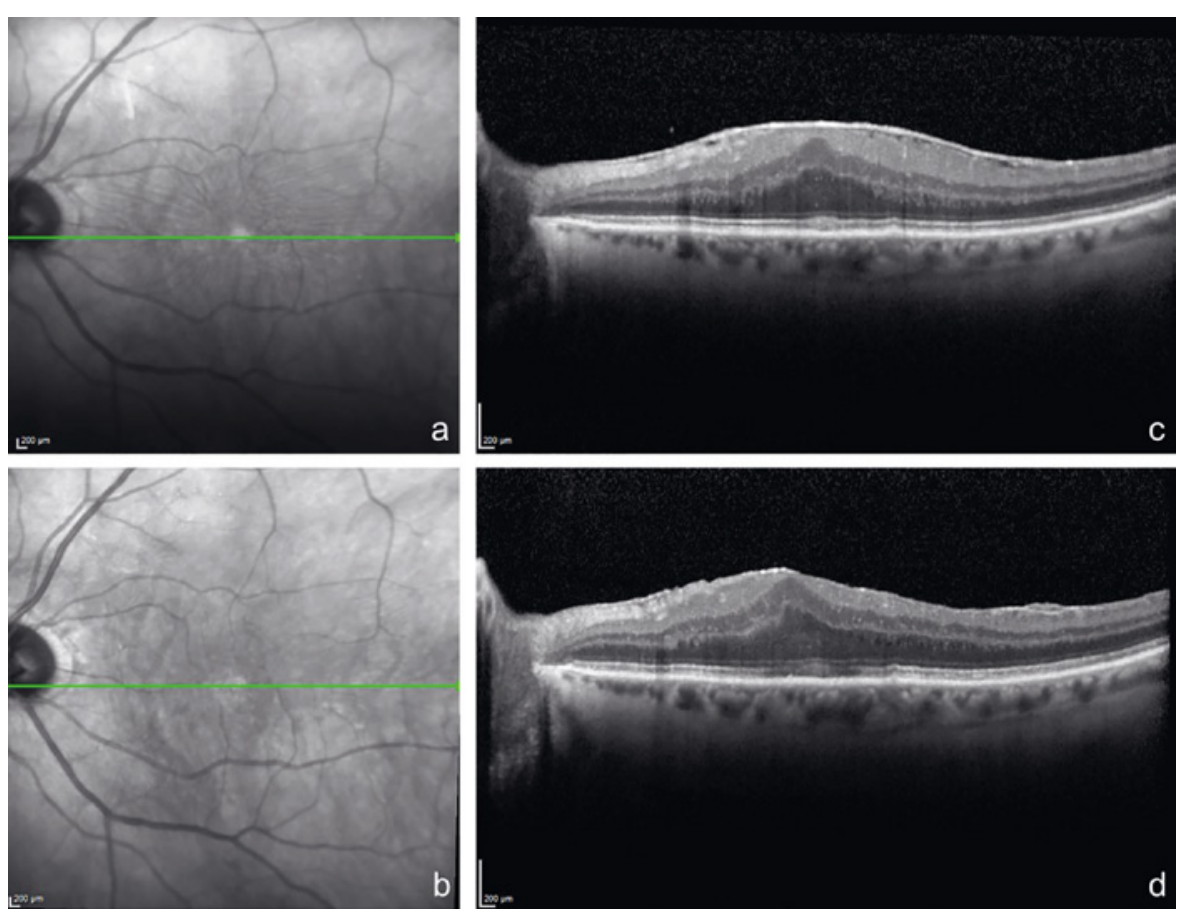
the one hand, lead to a better postoperative outcome and, on the other hand, provide objective outcome parameters which are correlated with visual acuity.

In particular, the inner retina irregularity index which, when determined preoperatively, allows statements to be made on the probable postoperative outcome, appears to be promising here. In connection with the increasing automation of image evaluation with the aid of "big data" and artificial intelligence, these results appear to be of particular interest.

\section{Disclosure Statement}

I hereby declare that there are no conflicts of interest with regard to this commentary.

\section{Republication}

This article was first published in Kompass Ophthalmol 2020;6:2628.

\section{References}

1 Bu S, Kuijer R, Li X, et al.: Idiopathic epiretinal membrane. Retina 2014;34: 2317-2335.

2 Maier M, Abraham S, Frank C, et al.: Therapie der vitreomakulären Traktion mit und ohne Makulaforamen. Klin Monatsbl Augenheilkd 2016;233:622630.

3 Duker J, Kaiser P, Binder S, et al.: The international vitreomacular traction study group classification of vitreomacular adhesion, traction, and macular hole. Ophthalmology 2013;120:2611-2619.

4 Jackson T, Nicod E, Simpson A, et al.: Symptomatic vitreomacular adhesion. Retina 2013;33:1503-1511.

5 Kim J, Kim D, Kim K, et al.: Visual prognostic factors of epiretinal membrane surgery in patients with pseudophakia. Ophthalmologica 2019;DOl: 10.1159/000502748.

Correspondence: Dr. Maximilian Treder, Klinik für Augenheilkunde, Universitätsklinikum Münster, Albert-Schweizer-Campus 1, 48149 Münster, Germany, maximilian.treder@ukmuenster.de 